\title{
A 16-Year old with Lemierre's Syndrome and Multiple Septic Pulmonary Emboli
}

\author{
Christopher M. Oermann ${ }^{1}$ \\ ${ }^{1}$ Children's Mercy Kansas City
}

September 22, 2020

A 16-Year old with Lemierre's Syndrome and Multiple Septic Pulmonary Emboli

Christopher M Oermann, MD

Department of Pediatrics, Children's Mercy Kansas City, Kansas City MO

Corresponding Author:

Christopher M Oermann, MD

Children's Mercy Kansas City

2401 Gillham Road

Kansas City, MO 64108

816-302-3354 (telephone)

816-302-9736 (fax)

cmoermann@cmh.edu

Running Head: Pulmonary Septic Emboli in Lemierre Sn

Key Words: Lemierre Syndrome, Septic Emboli

Word Count: 1035

To the Editor,

Lemierre's syndrome (LS) is a rare disease resulting from infective thrombophlebitis of the internal jugular vein (JV) following oropharyngeal infection. The incidence of LS has increased after decades of decline. Septic pulmonary emboli (PE) are common, but all organ systems can be involved. Although LS is most often caused byFusobacterium necrophorum, other pathogens have been implicated. Persistent high fever and organ-specific signs and symptoms result from the septic emboli (SE). The diagnosis is often suspected following growth of Fusobacterium from blood cultures and is confirmed by head/neck imaging. Therapy includes antimicrobials, drainage of abscesses, and anticoagulation. Significant morbidity/mortality occur if diagnosis and treatment are delayed. Pediatric pulmonologists must be familiar with the diagnosis and management of LS.

A previously healthy 16-year old male was admitted to hospital following two weeks of fever, myalgia, nausea/vomiting/diarrhea with 10-pound weight loss, and dry cough. He had been evaluated by his primary care provider (PCP) ten days prior to admission for pharyngitis and fever of $40^{\circ} \mathrm{C}$. Rapid streptococcal antigen and SARS-CoV-2 testing were negative, and he was treated with antipyretics. Continued symptoms led to PCP reevaluation after 7 days. Chest imaging demonstrated a single round pneumonia in the right 
lower lobe, and he received 3 days of azithromycin. He failed to improve and was referred to the emergency department 3 days later. Computed tomography (CT) of the chest/abdomen/pelvis demonstrated bilateral pulmonary nodules. He was admitted for evaluation and treatment.

Considerations from the admitting team included atypical infection (fungal or mycobacterial) versus ecigarette or vaping product use associated lung injury (EVALI), so Pulmonary Medicine service was consulted. EVALI was thought unlikely based on the radiographic appearance of the lesions (Figure 1). Additional considerations included septic PE, paragonimiasis, autoimmune disease with small vessel vasculitis (polyangiitis with granulomatosis), and inflammatory bowel disease. Evaluation for these potential diagnoses was initiated and empiric therapy with vancomycin and ceftriaxone was commenced. Echocardiogram, infectious diseases testing, and autoimmune evaluation were all normal. Painful swelling in the right, anterior neck and a tender spot on his upper right back developed the day after admission. His blood culture grew gram-negative anaerobic rods, raising concern for LS. Doppler ultrasound of the neck demonstrated occlusive thrombus within the right external JV with extension into the internal JV. Metronidazole was added to his intravenous antimicrobial regimen and anticoagulation therapy was initiated.

CT Imaging demonstrated normal CNS with confirmation of thrombosis of the JVs and inflammation over the surrounding soft tissues of the anterior right neck and a large abscess involving the posterior spinal musculature of the upper thorax. A drain was placed, and $25 \mathrm{cc}$ of pus removed. Magnetic resonance imaging of the spine demonstrated extension of the abscess into the $\mathrm{T} 1$ spinous process suggesting osteomyelitis. The blood culture grew Fusobacterium necrophorum and antibiotic coverage was changed to ampicillin/sulbactam to treat potential polymicrobial infection. Fevers resolved, pain and other symptoms improved, and the drain was removed after 3 days. He was discharged to continue 6 weeks of ampicillin/sulbactam and anticoagulation therapy.

LS is a rare complication of infections of the head/neck, with a reported incidence of one per million people per year. ${ }^{1}$ Common during the "pre-antibiotic era", LS had decreased in incidence for decades but has increased since the 1990s. This is possibly due to better antimicrobial stewardship and decreased use of antimicrobials for pharyngitis. ${ }^{1}$ Disease is attributed to infections of the tonsils and peritonsillar tissue in $87 \%$ of cases and infections of the pharynx, parotid glands, sinuses, mastoids, middle ears, and teeth/gums in $13 \% .^{2}$ Males and females are equally affected. ${ }^{3}$ LS has been reported in individuals aged 2 months to 78 years, although it is most commonly reported among previously healthy adolescents and young adults. ${ }^{2,3}$

LS originates with primary infection of the head/neck. ${ }^{4}$ This is followed by spread of the infection through the soft tissues of the neck resulting in thrombophlebitis of the internal JV. Dissemination of infection via SE occurs, with lung involvement in up to $80 \%$ and bones/joints in up to $27 \% .^{1-4}$ Additional sites of infection include cardiovascular, skin/muscles, central nervous system, abdominal organs (liver and spleen), and kidneys. Pulmonary involvement has included septic PE, abscess formation, necrotizing pneumonia, empyema, pneumothorax, pulmonary embolism, and acute respiratory distress syndrome. LS is most often caused by Fusobacterium necrophorum, part of the normal flora of the pharynx, accounting for up to 85\%. Additional causes include other Fusobacterium species, anaerobes (Bacteroides, Peptococcus, and Peptostreptococcusspecies), and aerobes (Streptococcus, and Staphylococcusspecies), among others. Polymicrobial infections are reported in up to $30 \%{ }^{2}$ Significant morbidity results from delayed diagnosis. Mortality has been reported as $4-18 \%$; more recent reviews suggest a lower rate. ${ }^{3,5}$

LS often presents 4-12 days after the initiating oropharyngeal infection, which may have resolved by presentation. Symptoms include high fever (up to 80\%), gastrointestinal (50\%), pharyngitis with cervical adenopathy or neck pain/swelling, myalgia/arthralgia, rigors, and respiratory symptoms (cough or dyspnea). ${ }^{1,2,5}$ Additional symptoms may be secondary to organ dysfunction caused by SE. Diagnosis is often made via Doppler ultrasound or CT imaging of the head/neck or the growth of Fusobacterium from blood/abscess cultures.

Therapy for LS involves long term, intravenous antibiotics, surgical drainage of abscesses, and anticoagulation. ${ }^{1-5}$ Metronidazole is often reported as standard therapy for LS. Other antibiotic consider- 
ations include carbapenems or penicillin/beta-lactamase inhibitor combinations, which provide broader coverage for polymicrobial infections. Duration of therapy is 4-6 weeks, allowing for adequate penetration of thrombi and treatment of secondary problems such as osteomyelitis. Anticoagulation therapy is more controversial; some data suggest that anticoagulation hastens overall response while others indicate adequate clinical response without additional therapy.

LS is a rare disorder of the head/neck that may be associated with significant morbidity/mortality if diagnosis and treatment are delayed. Pediatric pulmonologists will typically encounter LS in patients hospitalized with prolonged fever and multiple septic PE. LS must be considered in every child with multiple cavitary nodules identified on chest imaging. This is particularly true if there is a history of preceding oropharyngeal infection or signs/symptoms suggesting pathology in the head/neck. The growth of Fusobacterium species from blood culture or abscesses should also suggest LS. Doppler ultrasound examination of the neck and CT imaging of the head/neck should be obtained. Therapy is long-term, intravenous antibiotics. Adjuvant therapy includes surgical incision and drainage of abscesses and anticoagulation therapy.

\section{References}

1. Riordan T, Wilson M. Lemierre's syndrome: more than a historical curiosa. Postgrad Med J. 2004;80(944):328-334. doi:10.1136/pgmj.2003.014274

2. Ridgway JM, Parikh DA, Wright R, et al. Lemierre syndrome: a pediatric case series and review of literature. Am J Otolaryngol. 2010;31(1):38-45. doi:10.1016/j.amjoto.2008.09.006

3. Karkos PD, Asrani S, Karkos CD, et al. Lemierre's syndrome: A systematic review. Laryngoscope. 2009;119(8):1552-1559. doi:10.1002/lary.20542

4. Riordan T. Human infection with Fusobacterium necrophorum (Necrobacillosis), with a focus on Lemierre's syndrome. Clin Microbiol Rev. 2007;20(4):622-659. doi:10.1128/CMR.00011-07

5. Ouellette L, Barnes M, Flannigan M, Tavares E, Whalen D, Jones J. Lemierre's syndrome: A forgotten complication of acute pharyngitis. Am J Emerg Med. 2019;37(5):992-993. doi:10.1016/j.ajem.2018.09.049

Figure 1

Axial (1A) and coronal (1B) computed tomography images of the thorax. Images demonstrate multiple, bilateral, nodules with and without thick-walled cavitation. Most lesions are peripherally located; the lesions demonstrated on the coronal images are posteriorly located. 


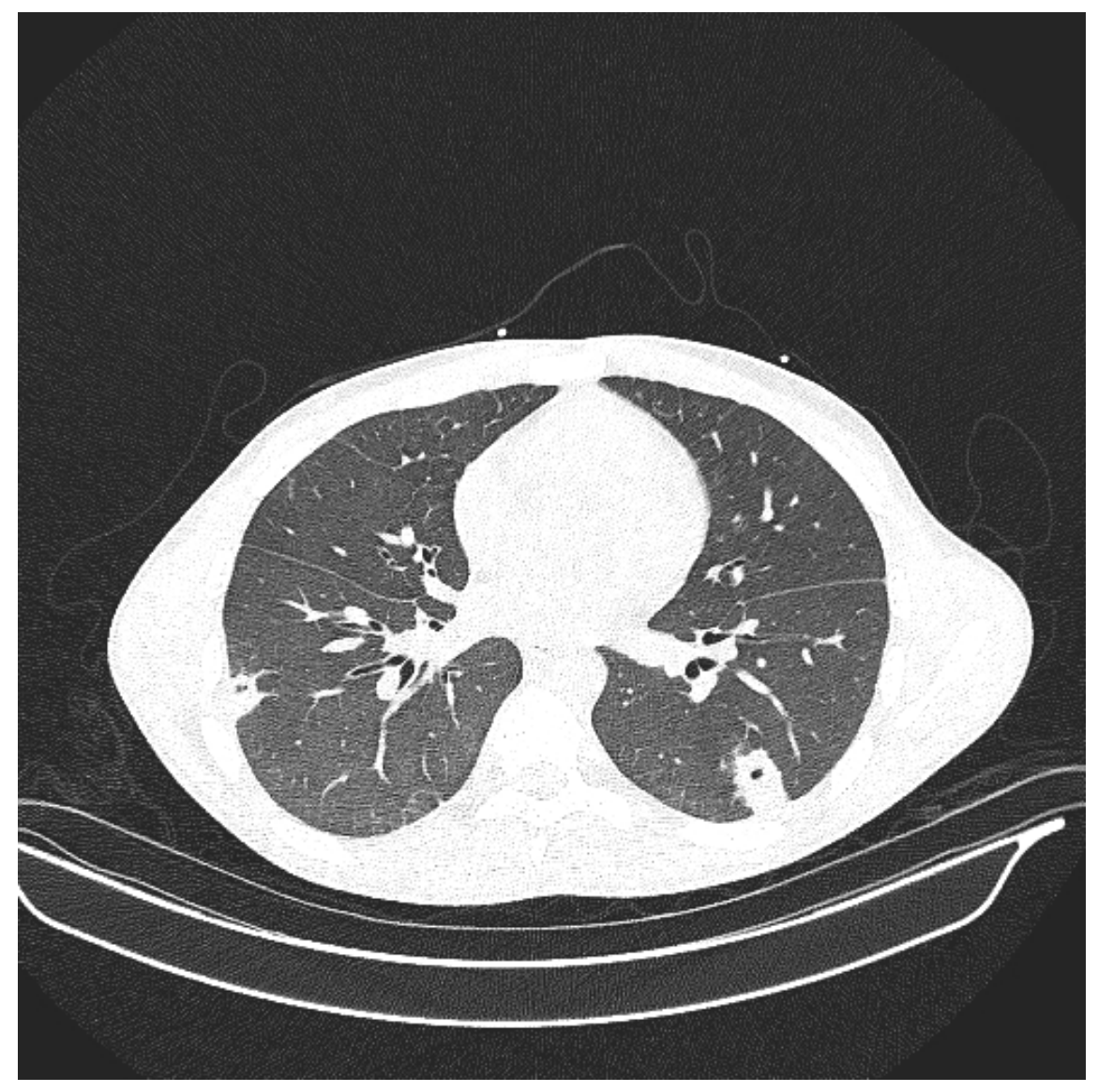



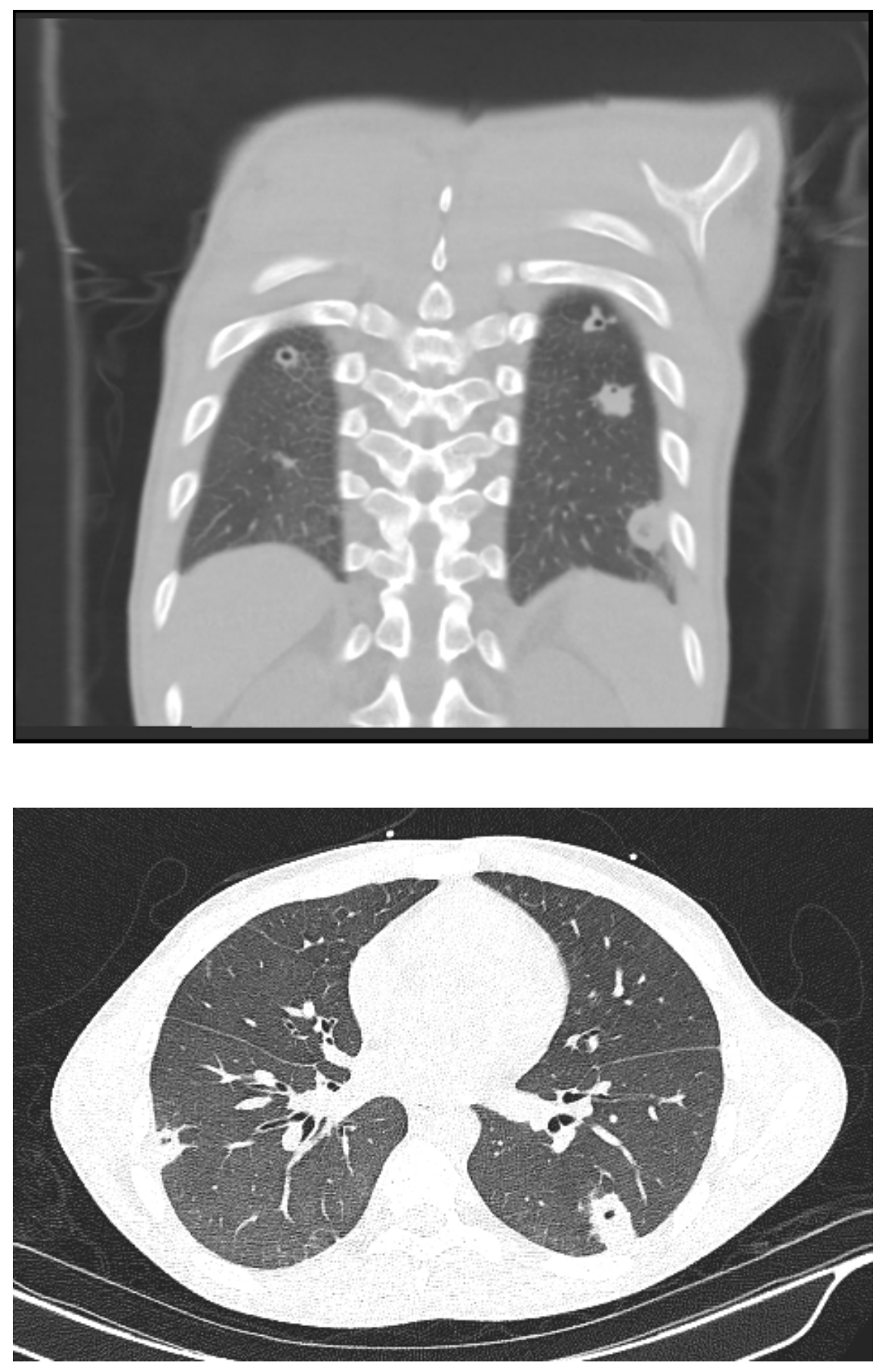


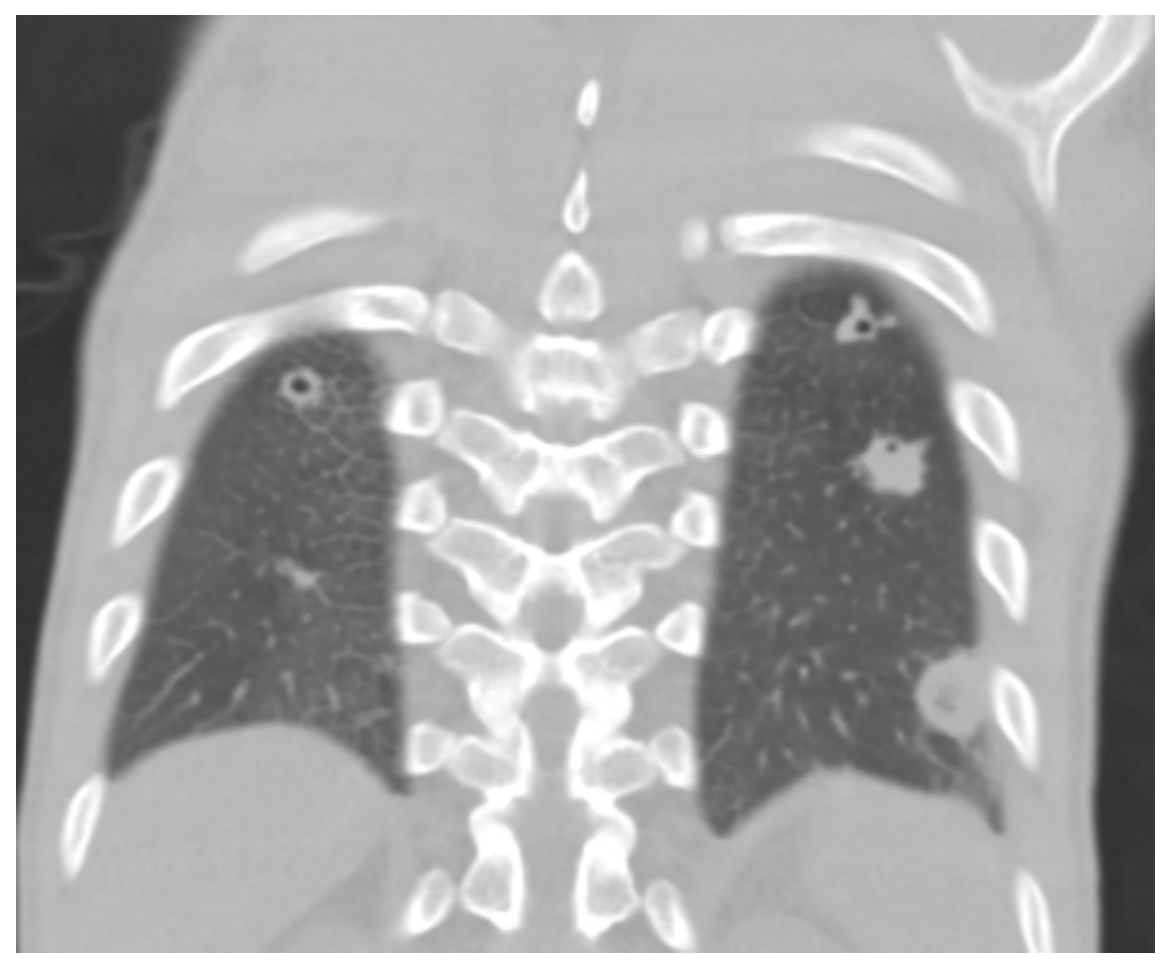

